João Marcos Feliciano de Souza

\title{
ANÁLISE DO ÍNDICE DE ASSINCRONIAS DURANTE A ASSISTÊNCIA VENTILATÓRIA NÃO INVASIVA COM AJUSTE NEURAL VERSUS VENTILAÇÃO NÃO INVASIVA CONVENCIONAL EM PEDIATRIA: uma revisão sistemática
}

Dissertação apresentada à Faculdade
Israelita de Ciências da Saúde Albert
Einstein para obtenção do Título de Mestre
em Ciências da Saúde.

São Paulo

2020 
João Marcos Feliciano de Souza

\title{
ANÁLISE DO ÍNDICE DE ASSINCRONIAS DURANTE A ASSISTÊNCIA VENTILATÓRIA NÃO INVASIVA COM AJUSTE NEURAL VERSUS VENTILAÇÃO NÃO INVASIVA CONVENCIONAL EM PEDIATRIA: uma revisão sistemática
}

\begin{abstract}
Dissertação apresentada à Faculdade Israelita de Ciências da Saúde Albert Einstein para obtenção do Título de Mestre em Ciências da Saúde.

Orientador: Prof. Dr. Eduardo Juan Troster Coorientadores: Prof. Dr. Celso Moura Rebello e Prof. Dr. Carlos Augusto Cardim de Oliveira
\end{abstract}


S729a

Souza, João Marcos Feliciano de Análise do índice de assincronias durante a assistência ventilatória não invasiva com ajuste neural versus ventilação não invasiva convencional em pediatria : uma revisão sistemática / João Marcos Feliciano de Souza. -- São Paulo, 2020.

viii, $19 \mathrm{f}$.

Dissertação (Mestrado) - Faculdade Israelita de Ensino e Pesquisa Albert Einstein. Instituto Israelita de Ensino e Pesquisa Albert Einstein. Programa de PósGraduação em Ciências da Saúde.

Título em inglês: Analysis of the asynchrony index during noninvasive neurally adjusted ventilatory assist versus conventional noninvasive ventilation in pediatrics: a systematic review.

1. Ventilação não invasiva. 2. Suporte ventilatório interativo. 3. Lactente. 4. Pré-escolar. 5. Criança.

NLM - WX 165

Elaborada pelo Sistema Einstein Integrado de Bibliotecas 
FACULDADE ISRAELITA DE CIÊNCIAS DA SAÚDE ALBERT EINSTEIN

Coordenador do Curso de Pós-Graduação: Prof. Dr. Luiz Vicente Rizzo 
João Marcos Feliciano de Souza

\title{
ANÁLISE DO ÍNDICE DE ASSINCRONIAS DURANTE A ASSISTÊNCIA VENTILATÓRIA NÃO INVASIVA COM AJUSTE NEURAL VERSUS VENTILAÇÃO NÃO INVASIVA CONVENCIONAL EM PEDIATRIA: Uma revisão sistemática
}

Presidente da banca: Prof. Dr. Eduardo Juan Troster

\author{
BANCA EXAMINADORA
}

Membros titulares:

Dra. Marina Buarque de Almeida

Dra. Graziela de Araujo Costa

Profa. Dra. Evelim Freitas Dantas Gomes

Membros suplentes:

Dra. Romy Schmidt Brock Zacharias

Dr. Nilton Ferraro de Oliveira

Aprovada em: 07/12/2020. 


\section{Sumário}

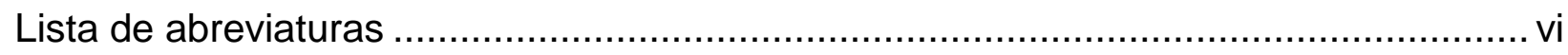

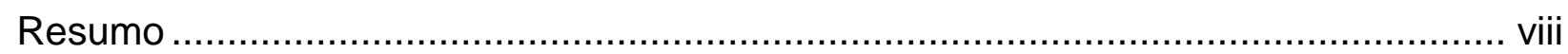

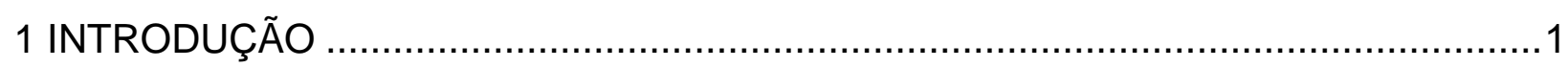

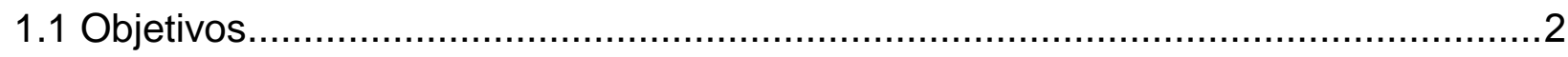

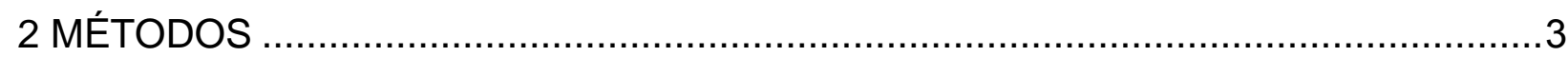

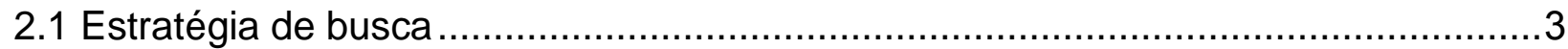

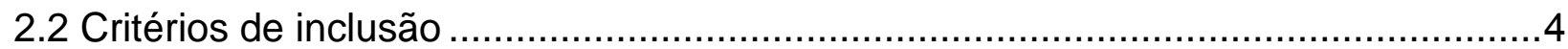

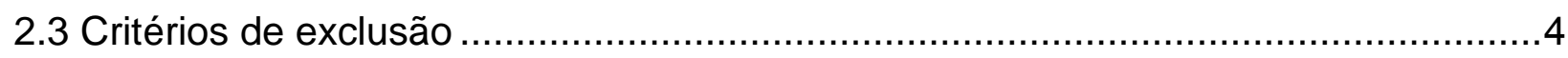

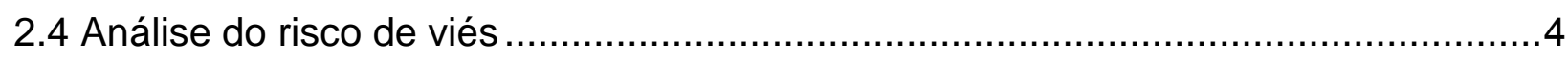

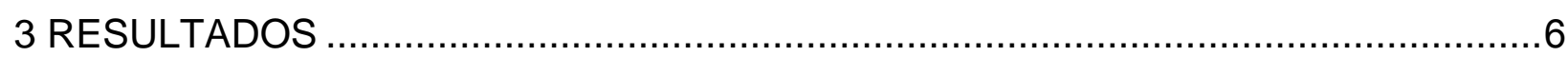

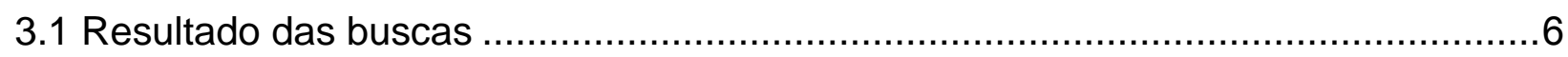

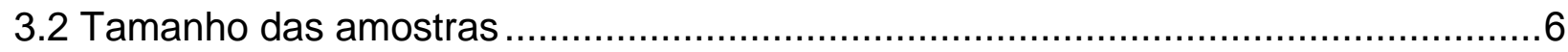

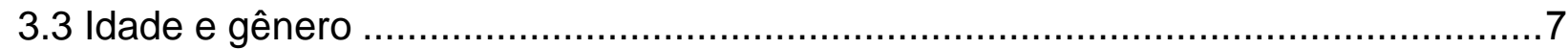

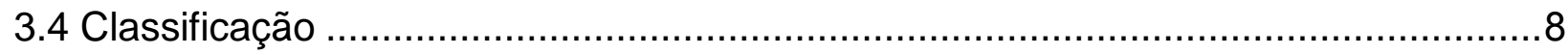

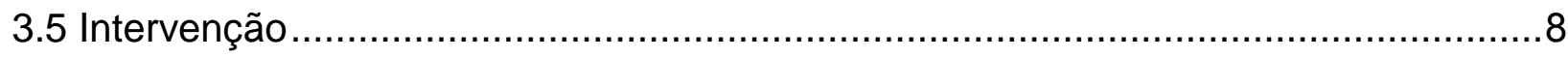

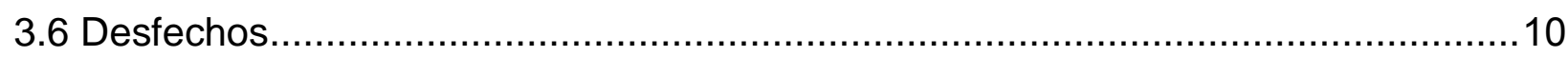

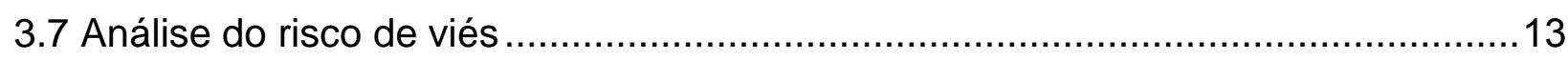

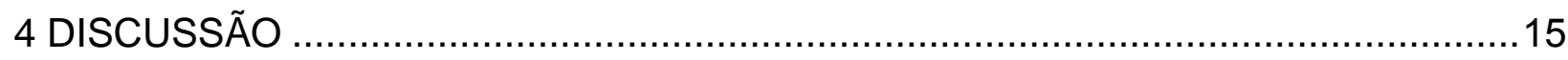

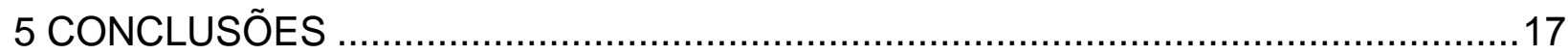

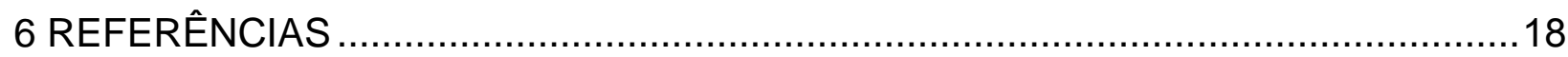

Abstract 


\section{Lista de abreviaturas}

AEdi Atividade elétrica do diafragma (Electrical activity of the diaphragm)

BVA Bronquiolite viral aguda

CP Ciclagem precoce

CRF Capacidade residual funcional

CT Ciclagem tardia

DA Disparo automático

DD Duplo disparo

El Esforço inefetivo

FC Frequência cardíaca

$\mathrm{FiO}_{2} \quad$ Fração inspirada de oxigênio

FR Frequência respiratória

IA Índice de assincronias (Asynchrony Index)

IIQ Intervalo interquartil

IRpA Insuficiência respiratória aguda (Acute respiratory failure)

MeSH Cabeçalhos de Assuntos Médicos (Medical Subject Headings)

$\mathrm{mmHg} \quad$ Milímetros de mercúrio

NCPAP Pressão positiva contínua nas vias aéreas nasais (Nasal continuous positive airway pressure)

NIPPV Ventilação nasal com pressão positiva intermitente (Nasal intermittent positive pressure ventilation)

NIV NAVA Assistência ventilatória não invasiva ajustada neuralmente (Noninvasive neurally adjusted ventilatory assist)

PAC Pressão assisto-controlada

$\mathrm{PaCO}_{2} \quad$ Pressão arterial de dióxido de carbono

$\mathrm{PaO}_{2} \quad$ Pressão arterial de oxigênio

PAV Pneumonia associada a ventilação mecânica

PEEP Pressão positiva expiratória final (Positive end-expiratory pressure)

$\mathrm{pH} \quad$ Potencial hidrogeniônico

PRISMA Itens de Relatório Preferidos para Revisões Sistemáticas e Meta-Análises (Preferred Reporting Items for Systematic Reviews and Meta-Analyses)

PS Pressão de suporte

RoB Risco de viés (Risk of bias) 
SGPP Sistema gerenciador de projeto de pesquisa

SNIPPV Ventilação nasal sincronizada com pressão positiva intermitente (Synchronized nasal intermittent positive pressure ventilation)

$\mathrm{SpO}_{2} \quad$ Saturação de oxigênio capilar periférico

$\mathrm{TcCO}_{2} \quad$ Dióxido de carbono transcutâneo

Te Tempo expiratório

Ti Tempo inspiratório

UTI Unidade de terapia intensiva (Intensive care unit)

VC Volume corrente

VM Ventilação mecânica (Mechanical ventilation)

VMI Ventilação mecânica invasiva

Vmin Volume minuto

VNI Ventilação não invasiva (Noninvasive ventilation) 


\section{Resumo}

Introdução: A insuficiência respiratória aguda é a condição mais prevalente em crianças internadas na unidade de terapia intensiva no Brasil e no mundo, sendo considerado o uso da ventilação mecânica não invasiva para o tratamento dos casos leves e moderados da doença. Os modos de disparo convencionais a fluxo ou pressão, durante a ventilação não invasiva, apresentam maior índice de assincronias quando comparados ao modo de disparo sincronizado com a atividade elétrica do diafragma. Com base em estudos prévios descritos na literatura, a hipótese do presente estudo é que há evidência de menor índice de assincronias e desfechos clínicos favoráveis relacionados ao modo non-invasive neurally adjusted ventilatory assist quando comparado ao uso da ventilação não invasiva convencional em pacientes pediátricos com insuficiência respiratória aguda. Objetivos: Comparar o índice de assincronias e os desfechos clínicos apresentados durante o uso da non-invasive neurally adjusted ventilatory assist versus ventilação não invasiva convencional no tratamento da insuficiência respiratória aguda na população pediátrica. Métodos: Trata-se de uma revisão sistemática de ensaios clínicos, realizada entre os meses de abril e maio de 2020 nas bases de dados eletrônicas Cochrane Library, Embase, LILACS, PubMed/MEDLINE, Scopus e Web of Science. Resultados: Foram encontrados 184 estudos, sendo quatro elegíveis para síntese qualitativa. A amostra analisou 39 participantes, com idade entre 35 dias e 15 anos com predomínio masculino $(61,5 \%)$. 0 desfecho primário analisado em três dos quatro estudos foi a diminuição significativa $(p<0,001)$ no índice de assincronias durante a non-invasive neurally adjusted ventilatory assist em comparação com a ventilação não invasiva convencional. Entretanto, a hipótese de relação entre a melhora da sincronia e desfechos clínicos favoráveis foi inconclusiva devido a limitações metodológicas dos estudos. Conclusões: A noninvasive neurally adjusted ventilatory assist diminui o índice de assincronias quando comparado a ventilação não invasiva convencional em pacientes pediátricos com insuficiência respiratória aguda. Entretanto, os demais desfechos clínicos apresentam resultados inconclusivos, necessitando de novos estudos com formatos metodológicos diferentes para sua confirmação.

PROSPERO ID: 181785

Descritores: Ventilação não invasiva; Suporte ventilatório interativo; Lactente; Préescolar; Criança 


\section{INTRODUÇÃO}

A insuficiência respiratória aguda (IRpA - acute respiratory failure) é a condição mais prevalente em crianças internadas na unidade de terapia intensiva (UTI - intensive care unit) no Brasil e no mundo. ${ }^{(1)}$ De acordo com a classificação de severidade e comprometimento do sistema respiratório, a ventilação não invasiva (VNI) será indicada como escolha primária no tratamento da IRpA. ${ }^{(2)}$

Os principais objetivos da VNI são: aumentar a capacidade residual funcional (CRF), diminuir o trabalho dos músculos respiratórios e melhorar significativamente a ventilação alveolar e a troca gasosa. ${ }^{(3-5)}$ Por se tratar de um tratamento não invasivo, a VNI previne e diminui a necessidade de intubação e reintubação traqueal, diminui o trabalho respiratório e ameniza as complicações causadas durante a ventilação mecânica invasiva (VMI), tais como: lesões traqueais e pneumonia associada a ventilação mecânica (PAV). ${ }^{(6)}$

A VNI Convencional dispõe de componentes pneumáticos que, ao detectar alterações de fluxo ou pressão, sincronizam os esforços respiratórios do paciente aos fluxos inspiratórios entregues. Desse modo, a escolha de interfaces com menores taxas de vazamento, está diretamente relacionada a melhor sincronia entre paciente e ventilador mecânico. ${ }^{(7)}$

Disponível apenas nos equipamentos da Getinge Group@, o modo de assistência ventilatória não invasiva ajustada neuralmente (NIV NAVA - noninvasive neurally adjusted ventilatory assist), utiliza eletrodos transesofágicos sensíveis a alterações da atividade elétrica do diafragma (AEdi - electrical activity of the diaphragm) e exclue o componente pneumático, sincronizando o fluxo inspiratório proporcionalmente aos esforços respiratórios. Ou seja, quanto maior a excitação diafragmática do paciente, maior será o pico de pressão de pico inspiratório entregue. ${ }^{(8)}$

A interação entre o paciente e o ventilador mecânico é avaliada por meio do índice de assincronias (IA - Asynchrony Index), obtido pela somatória dos eventos assincrônicos: esforços inefetivos (EI), disparo automático (DA), duplo disparo (DD), ciclagem precoce (CP) e ciclagem tardia (CT), dividida pela somatória dos ciclos respiratórios e El multiplicada por 100. O cálculo: $I A=[(E I+D A+D D+C P+C T) \div$ (ciclos respiratórios $+\mathrm{EI})] \times 100$, resultará em uma porcentagem de assincronia. ${ }^{(9,10)}$ Índices superiores ou iguais a $10 \%$ estão associados a desfechos clínicos 
desfavoráveis como por exemplo: alterações na entrega de volume corrente (VC), aumento do trabalho respiratório, maior necessidade do uso de sedativos, aumento do tempo em ventilação mecânica (VM - mechanical ventilation), aumento do tempo de permanência na UTI, além de maiores índices de mortalidade e lesão pulmonar induzida pela VM. ${ }^{(11,12)}$

Em virtude do que foi mencionado, o presente estudo apresenta duas possíveis hipóteses. A primeira é de que há evidências correlacionadas entre 0 uso da NIV NAVA e menores IA quando comparado a VNI Convencional em pacientes pediátricos com IRpA. A segunda é de que há evidências correlacionadas entre o uso da NIV NAVA e desfechos clínicos favoráveis quando comparado a VNI Convencional em pacientes pediátricos com IRpA.

Dessa forma, o objetivo dessa revisão sistemática foi analisar e comparar os eventos assincrônicos e os desfechos clínicos dos pacientes durante 0 uso do NIV NAVA versus VNI Convencional no tratamento da IRpA de crianças com idade entre 1 mês e 18 anos.

\subsection{Objetivos}

1. Comparar o Índice de assincronias durante o uso da assistência ventilatória não invasiva ajustada neuralmente (noninvasive neurally adjusted ventilatory assist) versus ventilação não invasiva convencional no tratamento da insuficiência respiratória aguda em pacientes pediátricos com idade entre 1 mês e 18 anos;

2. Comparar os desfechos clínicos durante o uso da assistência ventilatória não invasiva ajustada neuralmente versus ventilação não invasiva convencional no tratamento da insuficiência respiratória aguda em pacientes pediátricos com idade entre 1 mês e 18 anos. 


\section{MÉTODOS}

As diretrizes dos Itens de Relatório Preferidos para Revisões Sistemáticas e Meta-Análises (PRISMA - Preferred Reporting Items for Systematic Reviews and Meta-Analyses) foram utilizadas para delimitar os processos dessa revisão. Foram selecionados ensaios clínicos, que comparam o uso da NIV NAVA versus VNI Convencional no tratamento da IRpA de pacientes pediátricos, com faixa etária entre 1 mês a 18 anos de idade incompletos. O protocolo do estudo foi registrado na base de dados PROSPERO: International Prospective Register Of Systematic Reviews (ID: 181785) e no sistema gerenciador de projeto de pesquisa (SGPP) (ID: 4168-20).

Os autores declaram não haver uma fonte financiadora para o projeto, além de estarem isentos de qualquer tipo de conflito de interesse relacionado às marcas ou empresas citadas.

\subsection{Estratégia de busca}

As buscas foram realizadas por dois revisores independentes nas bases de dados eletrônicas da Cochrane Library, Embase, LILACS, PubMed/MEDLINE, Scopus e Web of Science entre os meses de abril e maio de 2020.

Utilizando os blocos temáticos: noninvasive ventilation, infant, child, preschool, child e neurally adjusted ventilatory assist, a pesquisa combinou os respectivos termos com operadores booleanos $O R$ e $A N D$ da seguinte forma: 'Noninvasive Ventilations' OR 'Ventilation, Noninvasive' OR 'Ventilations, Noninvasive' $O R$ 'Non-Invasive Ventilation' OR 'Non-Invasive Ventilations' $O R$ 'Ventilation, NonInvasive' OR 'Ventilations, Non-Invasive' OR 'Non Invasive Ventilation' OR 'Non Invasive Ventilations' OR 'Ventilation, Non Invasive' OR 'Ventilations, Non Invasive'; AND 'Children' OR 'Preschool Child' OR 'Children, Preschool' OR 'Preschool Children Adolescent' OR 'Child'; AND 'Support, Interactive Ventilatory' OR 'Ventilatory Support, Interactive' OR 'Neurally Adjusted Ventilatory Assist'. 


\subsection{Critérios de inclusão}

A análise primária dos artigos foi feita após leitura do título e do resumo, aplicando os seguintes critérios para inclusão: 1. Ensaios clínicos randomizados ou não randomizados; 2 . Faixa etária dos sujeitos de pesquisa entre 1 mês e 18 anos incompletos; 3. Pacientes com insuficiência respiratória; 4. Em uso dos seguintes modos de VNI: (a) Ventilação nasal com pressão positiva intermitente (NIPPV - nasal intermittent positive pressure ventilation), (b) Ventilação nasal sincronizada com pressão positiva intermitente (SNIPPV - Synchronized nasal intermittent positive pressure ventilation) ou NIV NAVA.

\subsection{Critérios de exclusão}

Não havendo restrições quanto ao tipo de idioma e ano de publicação, foram aplicados os seguintes critérios para exclusão: 1. Estudos observacionais retrospectivos e prospectivos; 2. Estudos em modelos experimentais não humanos; 2. Publicações sem os resultados disponíveis na íntegra para análise individual dos sujeitos de pesquisa; 3. Estudos em populações compostas exclusivamente por recém-nascidos ou adultos; 4. Pacientes em uso de VNI por modo de pressão positiva contínua nas vias aéreas nasais (NCPAP - nasal continuous positive airway pressure); 7. Pacientes em uso de VM invasiva e 8. Estudos interrompidos ou em andamento.

\subsection{Análise do risco de viés}

Por meio da ferramenta risco de viés (RoB - risk of bias) da Cochrane Library, foi possível avaliar a qualidade metodológica e a validade interna dos estudos. Classificando-os quanto ao alto, baixo ou risco incerto de viés, foram aplicados os seguintes critérios: 1. Geração da sequência de randomização; 2. Sigilo da alocação; 3. Ocultamento da alocação; 4. Mascaramento (cegamento) dos participantes e da equipe de pesquisadores; 5. Mascaramento (cegamento) na 
avaliação dos desfechos; 6. Dados incompletos dos desfechos; 7. Relato seletivo dos desfechos e 8. Outras fontes de viés identificadas pelo revisor. ${ }^{(13)}$ 


\section{RESULTADOS}

\subsection{Resultado das buscas}

A pesquisa bibliográfica realizada entre abril e maio de 2020 resultou na identificação de 184 artigos. Sendo 69 obtidos na base de dados eletrônica Cochrane Library, 41 na Embase, 22 na LILACS, 22 na PubMed/MEDLINE, cinco na Scopus e 25 na Web of Science.

Após a remoção de 58 artigos duplicados, outros 122 foram excluídos por não atenderem aos critérios de inclusão durante a análise do título e do resumo conforme observado na figura 1. Desses, três estudos constam como interrompidos, um estudo apresenta status "unknow" referente a não atualização de dados pelos autores por um período superior a dois anos, enquanto um ECR comparativo entre NIV NAVA e VNI convencional com uma amostra estimada de 14 participantes prematuros consta com status "recruiting" na plataforma ClinicalTrials.gov.

O processo de seleção incluiu quatro artigos para a análise qualitativa (Tabela 1). Sendo dois ensaios clínicos randomizados ${ }^{(14,15)}$ e dois ensaios clínicos não randomizados. ${ }^{(16,17)}$

\subsection{Tamanho das amostras}

O tamanho das amostras dos quatro artigos variou entre seis a 18 participantes, ${ }^{(14,15)}$ disponibilizando os resultados de 48 participantes no total.

Durante a análise individual dos sujeitos de pesquisa, nove $(18,75 \%)$ foram excluídos das análises por não atenderem aos critérios de inclusão de faixa etária (menor que 1 mês) e modo ventilatório (NCPAP). Dessa forma, o total de 39 $(81,25 \%)$ sujeitos foram efetivamente incluídos na análise qualitativa (Tabela 1). 


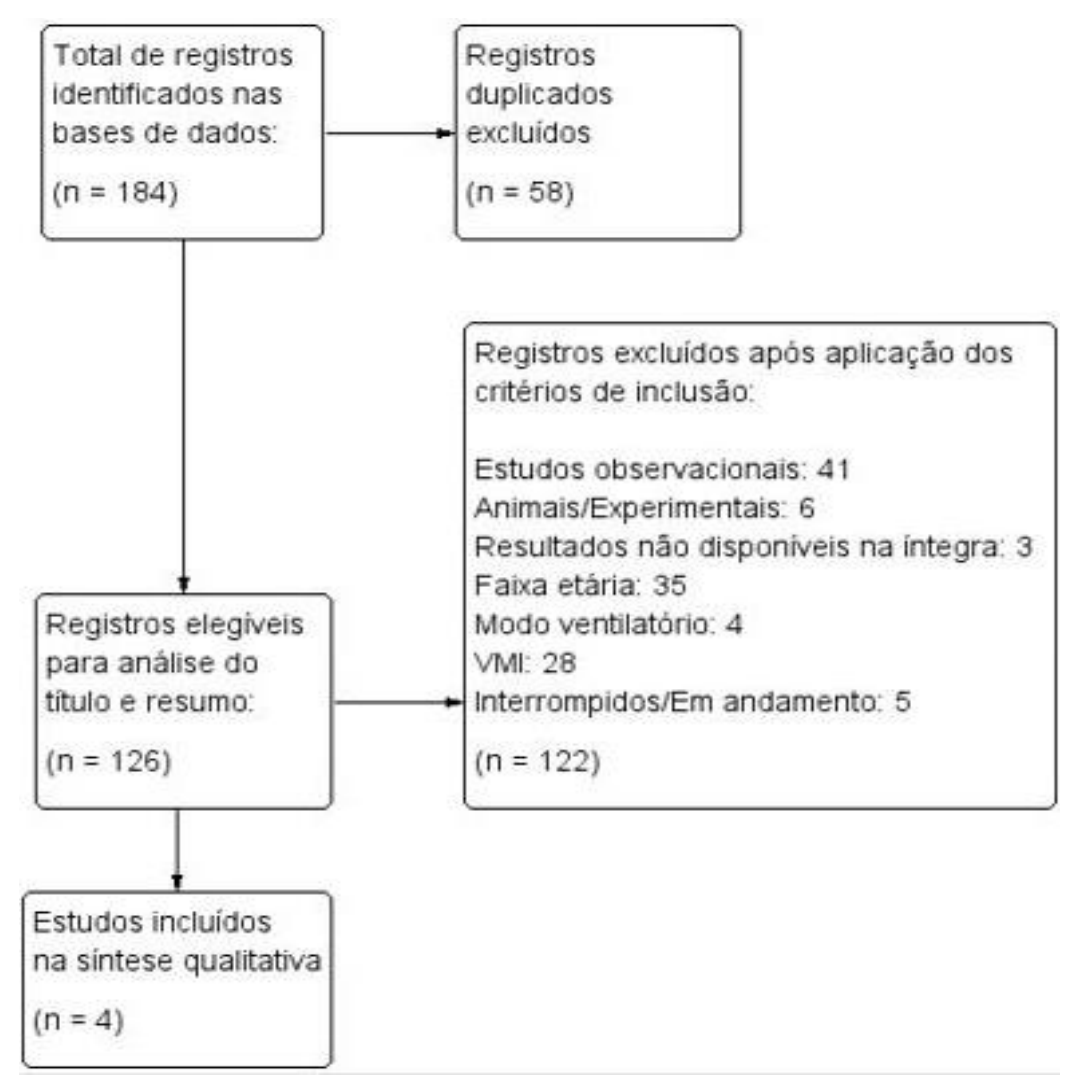

Figura 1. Diagrama de fluxo da pesquisa

\subsection{Idade e gênero}

A idade dos sujeitos elegíveis variou entre 35 dias de vida a 15 anos e 10 meses, com predomínio masculino variando entre $66 \%$ a $80 \%$ (Tabela 1). A análise do estudo de Vignaux et al. (2013), apresentou uma mediana de idade de 18 meses da amostra total $(n=6)$, com intervalo interquartil (IIQ) $1=5-I I Q 3=27$. Além de predomínio masculino em $66 \%{ }^{(14)}$

No estudo de Baudin et al. (2014), a mediana de idade do total da amostra $(n=11)$ foi de 0,9 meses $(0,53-1,7)$. Entretanto seis sujeitos $(54 \%)$, foram excluídos da análise por não atenderem os critérios de inclusão por faixa etária. Dessa forma a mediana de idade da amostra elegível $(n=5)$ passa a ser de 1,87 meses $(1,53$ $2,1)$, com predomínio masculino em $80 \%$. $^{(16)}$

No estudo de Ducharme-Crevier et al. (2015), a mediana de idade do total da amostra $(n=13)$ foi de 42 meses (2 - 76). Entretanto três sujeitos (23\%), foram excluídos da análise por não atenderem aos critérios de inclusão de modo ventilatório (NCPAP). Dessa forma a mediana de idade da amostra elegível $(n=10)$ passa a ser de 53,75 meses $\left(16,5\right.$ - 125,5), com predomínio masculino em $70 \%$. $^{(17)}$ 
No estudo de Chidini et al. (2016), a mediana de idade do total da amostra $(n=18)$ foi de 13 meses $(8,5-17,75)$, sem predomínio de gênero. ${ }^{(15)}$

Tabela 1. Características das amostras

\begin{tabular}{lccccc}
\hline Autor/Ano & $\begin{array}{c}\text { Sequência de } \\
\text { intervenção }\end{array}$ & $\begin{array}{c}\text { Amostr } \\
\text { a total } \\
(\mathbf{n = 4 8 )}\end{array}$ & $\begin{array}{c}\text { Amostra } \\
\text { elegível } \\
(\mathbf{n = 3 9 )}\end{array}$ & $\begin{array}{c}\text { Mediana de idade } \\
\text { (meses) da } \\
\text { amostra elegível } \\
\text { (IIQ1-IIQ3) }\end{array}$ & $\begin{array}{c}\text { Gênero } \\
\text { masculino } \\
\text { (\%) }\end{array}$ \\
\hline $\begin{array}{l}\text { Vignaux et al. 2013(14) } \\
\text { Chidini et al. 2016 }{ }^{(15)}\end{array}$ & Randomizada & 6 & 6 & $18(5-27)$ & 66 \\
$\begin{array}{l}\text { Baudin et al. 2014 } \\
\text { Ducharme-Crevier et }\end{array}$ & Não randomizada & 11 & 5 & $1,87(1,53-2,1)$ & 80 \\
al. 2015 & Não randomizada & 13 & 10 & $53,75(16,5-125,5)$ & 70 \\
\hline
\end{tabular}

\subsection{Classificação}

Todos os estudos classificaram os sujeitos com base na IRpA durante o período de internação na UTI pediátrica.

A relação entre a pressão arterial de oxigênio e fração inspirada de oxigênio $\left(\mathrm{PaO}_{2} / \mathrm{FiO}_{2}\right)$ menor que 300 , frequência respiratória $(\mathrm{FR})$ acima do valor predito para a idade, uso de musculatura respiratória acessória e dificuldade na alimentação, foram os critérios de classificação utilizados por Chidini et al. (2016). ${ }^{(15)}$

$\mathrm{O}$ uso de $\mathrm{VNI}$ de forma contínua durante um período maior que $6 \mathrm{~h}$ foi um dos critérios de classificação no estudo de Ducharme-Crevier et al. (2015). ${ }^{(17)} \mathrm{A}$ falha terapêutica no uso do NCPAP, eventos recorrentes de apneia em um intervalo menor que $1 \mathrm{~h}$, sinais de desconforto respiratório com alterações gasométricas como: potencial hidrogeniônico $(\mathrm{pH})<7,30$ e pressão arterial de dióxido de carbono $\left(\mathrm{PaCO}_{2}\right)$ maior que 60 milímetros de mercúrio $(\mathrm{mmHg})$ foram os critérios de classificação adotados por Baudin et al. (2014). ${ }^{(16)}$ Já Vignaux et al. (2013) aplicaram a VNI após a extubação de pacientes intubados para realização de procedimentos cirúrgicos. ${ }^{(14)}$

\subsection{Intervenção}

De acordo com o método de seleção cruzada (cross-over) adotado pelos autores, todos os sujeitos participaram simultaneamente do grupo NIV NAVA e VNI Convencional (Tabela 2). 
O período de análise dos desfechos em cada grupo variou entre 10 a 60 minutos. Sendo que o intervalo de tempo entre as intervenções, denominado "período de washout", não foi descrito nos estudos de Ducharme-Crevier et al. (2015), ${ }^{(17)}$ e variou entre cinco e 70 minutos nos estudos de Vignaux et al. (2013), Baudin et al. (2014) e Chidini et al. (2016). ${ }^{(14-16)}$

Durante a NIV NAVA foi utilizado apenas um dispositivo (Servo i® Getinge Group(), e durante a VNI Convencional foi descrito o uso de cinco dispositivos diferentes (Babylog® 8000 plus - Draeger; Servo $i \circledast$ - Getinge Group@) VPAP III ResMed; BIPAP $\AA^{\text {e Trilogy } 100 ® ~-~ P h i l i p s) . ~ C i n c o ~ t i p o s ~ d e ~ i n t e r f a c e s ~ d e ~ V N I ~ t a m b e ́ m ~}$ foram descritos (máscara facial total, máscara oronasal, máscara nasal, pronga nasal, e tubo nasofaríngeo).

Vignaux et al. de 2013 aplicaram de forma randomizada, um total de 25 minutos de VNI Convencional, pressão de suporte (PS) seguidos por 20 minutos de NIV NAVA. Foi utilizado o mesmo dispositivo (Servo i® - Getinge Group@) com um período de washout mínimo de cinco minutos entre as intervenções. Enquanto as interfaces (máscara facial e pronga nasal) podiam variar conforme a tolerância e agitação do participante. ${ }^{(14)}$

Baudin et al. (2014) aplicaram uma sequência não randomizada de 2 horas em VNI Convencional, pressão assisto-controlada (PAC) seguido de 2 horas em NIV NAVA. Foi utilizado o mesmo dispositivo (Servo $i \AA$ - Getinge Group@) e a mesma interface (máscara nasal) em ambas as intervenções. Apenas os últimos 10 minutos de cada período foram gravados e utilizados na comparação de assincronias, promovendo um washout de 110 até 130 minutos entre os dados gravados. ${ }^{(16)}$

Ducharme-Crevier et al. (2015) aplicaram uma sequência não randomizada composta por três períodos. Inicialmente 30 minutos em VNI Convencional (NCPAP, PAC ou PS), seguido de 60 minutos em NIV NAVA, e por fim mais 30 minutos em VNI Convencional. Diversos tipos de dispositivos (Babylog $8000 \AA$ - Draeger; Servo i® - Getinge Group@; VPAP III® - ResMed; BIPAP® e Trilogy $100 \AA$ Philips) e interfaces (tubo nasofaríngeo, máscara nasal e máscara nasobucal) foram utilizados. Os últimos 10 minutos de cada período foram utilizados na comparação das assincronias, sem que houvesse um período específico de washout entre as intervenções. ${ }^{(17)}$

Chidini et al. (2016) aplicaram de forma randomizada, 60 minutos de VNI Convencional (PS) e 60 minutos de NIV NAVA, com um período de washout de 20 
minutos. Foi utilizado o mesmo dispositivo (Servo iß - Getinge Group@) e a mesma interface (máscara facial total) em ambas as intervenções. ${ }^{(15)}$

Tabela 2. Dados metodológicos dos estudos selecionados

\begin{tabular}{|c|c|c|c|c|c|c|}
\hline Autor/Ano & $\begin{array}{c}\text { Amostra } \\
\text { total } \\
(n=48)\end{array}$ & Dispositivo & Interface & $\begin{array}{c}\text { Período de } \\
\text { washout } \\
\text { (minutos) }\end{array}$ & $\begin{array}{c}\text { Período } \\
\text { em NIV } \\
\text { NAVA } \\
\text { (minutos) } \\
\end{array}$ & $\begin{array}{c}\text { Período em } \\
\text { VNI } \\
\text { convencional } \\
\text { (minutos) }\end{array}$ \\
\hline $\begin{array}{l}\text { Vignaux } \\
\text { et al.2013 }\end{array}$ & 6 & Servo i® & $\begin{array}{c}\text { Máscara } \\
\text { facial ou } \\
\text { pronga nasal }\end{array}$ & $>5$ & 20 & 25 \\
\hline $\begin{array}{l}\text { Chidini } \\
\text { et al.2016 }\end{array}$ & 18 & Servo $i \AA$ & $\begin{array}{l}\text { Máscara } \\
\text { facial total }\end{array}$ & 20 & 60 & 60 \\
\hline $\begin{array}{l}\text { Baudin } \\
\text { et al.2014 }{ }^{(16)}\end{array}$ & 11 & Servo iß & $\begin{array}{c}\text { Máscara } \\
\text { nasal }\end{array}$ & $110-130$ & 10 & 10 \\
\hline $\begin{array}{l}\text { Ducharme- } \\
\text { Crevier et } \\
\text { al.2015 }\end{array}$ & 13 & $\begin{array}{c}\text { Servo i® } \\
+ \\
\text { outros* }^{*}\end{array}$ & Diversas** & $\begin{array}{l}\text { Não há } \\
\text { descrição }\end{array}$ & 10 & 20 \\
\hline
\end{tabular}

${ }^{*}$ Babylog $8000 \AA$ - Draeger, VPAP III® - ResMed, BIPAP® e Trilogy $100 \AA$ - Philips. ${ }^{* \star}$ Tubo nasofaríngeo, máscara nasal e máscara nasobucal.

\subsection{Desfechos}

O desfecho primário observado em três dos quatro artigos foi o IA ou Asynchrony Index (AI) (Tabela 3). Foi possível observar uma diminuição significativa do IA durante a NIV NAVA quando comparada a VNI Convencional $(p<0,001) .{ }^{(14-16)}$

No estudo de Ducharme-Crevier et al. (2015) os desfechos relacionados a sincronia foram quantificados de acordo com a porcentagem de tempo de VM assincrônica e não pelo IA. Foi possível observar uma diminuição na porcentagem de tempo de VM assincrônica durante a NIV NAVA quando comparada a VNI Convencional $(p=0,05)$. Os autores definiram como desfecho primário a viabilidade e a tolerância da NIV NAVA. Concluindo que seu uso foi viável, de acordo com sua capacidade em obter um sinal correto da AEdi, em diminuir a porcentagem de tempo de VM em assincronia e pela não interrupção do suporte ventilatório, além de ser tolerável por não apresentar diferenças nas condições clínicas dos pacientes internados na UTI. ${ }^{(17)}$

Outros desfechos como a média dos parâmetros ventilatórios, características clínicas, trocas gasosas e uso de sedativos também foram quantificados (Tabela 3), apresentando aumento significante nos valores de: pressão de pico inspiratória $(p=0,03)$ e pressão média das vias aéreas $(p=0,016)$ durante a NIV NAVA. 
Não houve alterações significantes nos valores de FR ( $p=0,37)$, VC $(p=0,562)$, pico de AEdi $(p=0,077)$, tempo inspiratório (Ti) neural $(p=0,95)$ e porcentagem de vazamentos $(p=0,817)$ entre as intervenções nos estudo de Vignaux et al. (2013). ${ }^{(14)}$

Os estudos de Baudin et al. (2014), apresentaram aumento significante da FR do VM $(p=0,03)$ durante a NIV NAVA. Não houve alterações significantes nos valores de pressão de pico inspiratória $(p=0,09), \mathrm{FiO}_{2}(p=0,22)$, pressão positiva expiratória final (PEEP - positive end-expiratory pressure) ( $p=0,95)$, FR neural $(p=0,16)$, pico de AEdi $(p=0,80)$, saturação de oxigênio capilar periférico $\left(\mathrm{SpO}_{2}\right)(\mathrm{p}=0,07)$, frequência cardíaca $(\mathrm{FC})(\mathrm{p}=0,18)$, dióxido de carbono transcutâneo $\left(\mathrm{TcCO}_{2}\right)(p=0,3)$ e desconforto respiratório $(\mathrm{p}=0,87)$ entre as intervenções. ${ }^{(16)}$

Os estudos de Chidini et al. (2016), apresentaram alterações significantes na diminuição do Índice de Oxigenação (IO) $(p=0,043)$, pico de fluxo inspiratório $(p=0,001)$, pressão de pico inspiratória $(p=0,006)$ e pressão média das vias aéreas $(p=0,038)$, além de aumento do volume minuto $(V \min )(p=0,038)$ e FR neural $(\mathrm{p}=0,013)$ durante a NIV NAVA. Não houve alterações significantes nos valores de $\mathrm{pH}$, $\mathrm{PaCO}_{2}, \mathrm{PaO}_{2} / \mathrm{FiO}_{2}$, uso de sedativos $(\mathrm{p}=0,752), \mathrm{FiO}_{2}(\mathrm{p}=0,871), \mathrm{VC}(\mathrm{p}=0,631), \mathrm{Ti}$ neural $(p=0,051)$, tempo expiratório $(T e)$ neural $(p=0,657)$ e pico de AEdi $(p=0,013)$ entre as intervenções. ${ }^{(15)}$ 
Tabela 3. Análise descritiva dos estudos selecionados

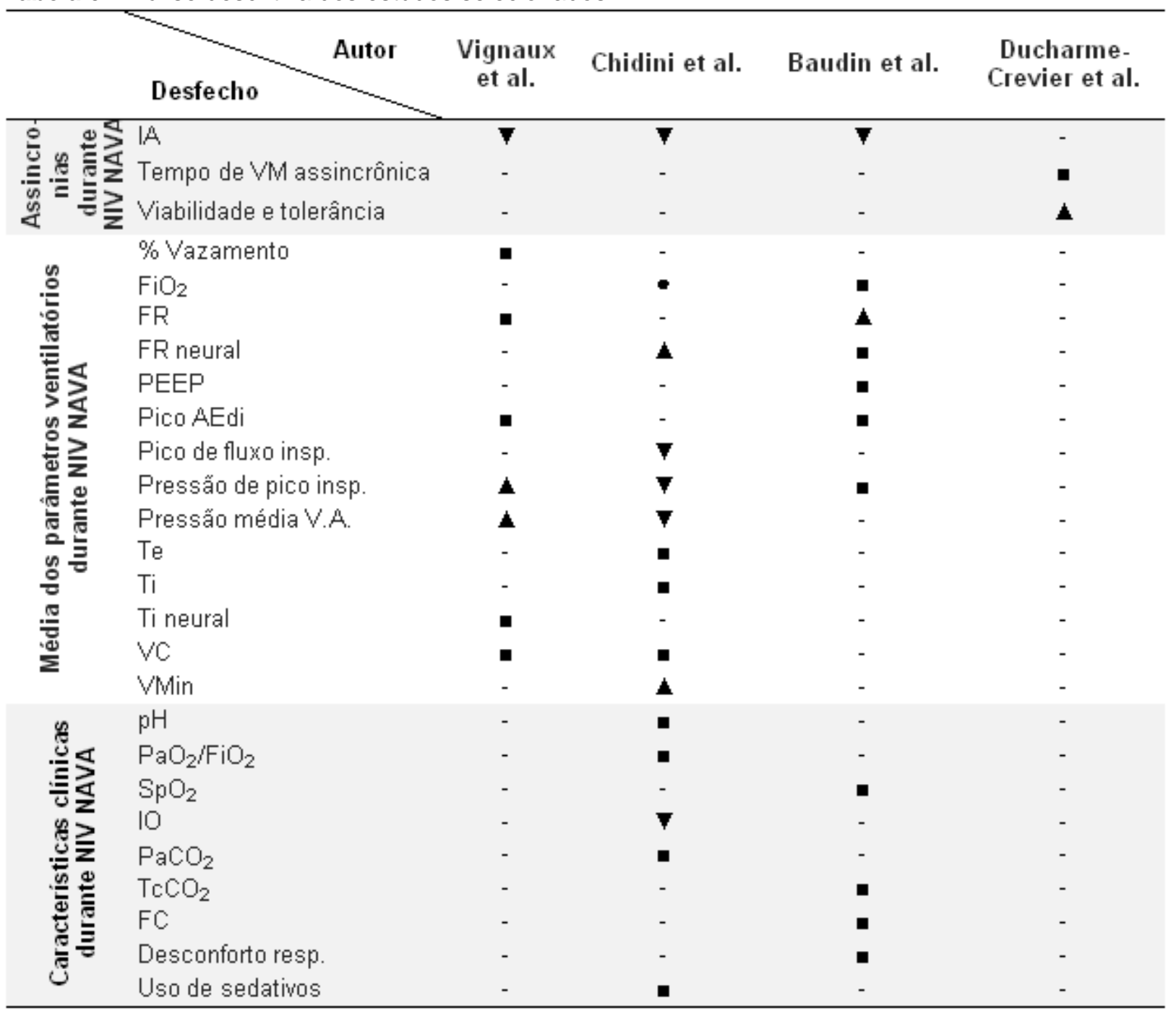

Diminuição significante $(p<0,05)$; $\boldsymbol{\Delta}$ Aumento significante $(p<0,005)$; I Sem alterações significantes $(p \geq 0,05)$. AEdi: atividade elétrica do diafragma; $\mathrm{FC}$ : frequência cardíaca; $\mathrm{FiO}_{2}$ : fração inspirada de oxigênio; FR: frequência respiratória; IA: índice de assincronias; IO: índice de oxigenação; NIV NAVA: assistência ventilatória não invasiva ajustada neuralmente (noninvasive neurally adjusted ventilatory assist); $\mathrm{PaCO}_{2}$ : pressão arterial de dióxido de carbono; $\mathrm{PaO}_{2}$ : pressão arterial de oxigênio; PEEP: pressão positiva expiratória final (positive end-expiratory pressure); $\mathrm{pH}$ : potencial hidrogeniônico; $\mathrm{SpO}_{2}$ : saturação de oxigênio capilar periférico; $\mathrm{TcCO}_{2}$ : dióxido de carbono transcutâneo; Te: tempo expiratório; Ti: tempo inspiratório; VC: volume corrente; VM: ventilação mecânica; Vmin: volume minuto.

Fonte: Vignaux L, Grazioli S, Piquilloud L, Bochaton N, Karam O, Levy-Jamet Y, et al. Patient-ventilator asynchrony during noninvasive pressure support ventilation and neurally adjusted ventilatory assist in infants and children. Pediatr Crit Care Med. 2013;14(8):e357-64. ${ }^{(14)}$

Chidini G, De Luca D, Conti G, Pelosi P, Nava S, Calderini E. Early noninvasive neurally adjusted ventilatory assist versus noninvasive flow-triggered pressure support ventilation in pediatric acute respiratory failure: a physiologic randomized controlled trial. Pediatr Crit Care Med. 2016;17(11):e48795. ${ }^{(15)}$

Baudin F, Pouyau R, Cour-Andlauer F, Berthiller J, Robert D, Javouhey E. Neurally adjusted ventilator assist (NAVA) reduces asynchrony during non-invasive ventilation for severe bronchiolitis. Pediatr Pulmonol. 2015;50(12):1320-7. ${ }^{(16)}$

Ducharme-Crevier L, Beck J, Essouri S, Jouvet P, Emeriaud G. Neurally adjusted ventilatory assist (NAVA) allows patient-ventilator synchrony during pediatric noninvasive ventilation: a crossover physiological study. Crit Care. 2015;19(1):44. ${ }^{(17)}$ 


\subsection{Análise do risco de viés}

A análise de qualidade metodológica dos estudos, observados nas figuras 2 e 3, foi obtida utilizando os critérios de avaliação da ferramenta RoB. ${ }^{(13)}$ Com exceção do estudo publicado por Chidini et al. (2016), ${ }^{(15)}$ todos classificaram ao menos um item como sendo de "alto risco" ou "risco incerto" de viés metodológico.

A não randomização das intervenções aplicadas por Baudin et al. (2014) e Ducharme-Crevier et al. (2015), apresentam um "alto risco" de viés metodológico. ${ }^{(16,17)}$

O não cegamento na alocação dos sujeitos nos estudos publicados por Baudin et al. (2014) e Ducharme-Crevier et al. (2015) apresentam um "alto risco" de viés. ${ }^{(16,17)}$ Enquanto a não descrição desse item no estudo publicado por Vignaux et al. (2013), o classifica como "risco incerto" de viés. ${ }^{(14)}$

Em nenhum dos quatro artigos analisados houve cegamento dos sujeitos ou da equipe de pesquisa. Segundo o julgamento dos autores desta revisão, esse item não apresentou interferências na análise dos resultados. Sendo assim, os estudos são classificados como "baixo risco" de viés. ${ }^{(14-17)}$

O cegamento durante a avaliação dos desfechos foi descrito nos estudos de Baudin et al. (2014) e Chidini et al. (2016), e foi classificado como "baixo risco" de viés. ${ }^{(15,16)}$ A não descrição desse item nos estudos de Vignaux et al. (2013) e Ducharme-Crevier et al. (2015) foi classificada como "risco incerto" de viés. ${ }^{(14,17)}$

Todos os estudos aparentam estar livres de vieses de atrito e de comunicação, relacionados respectivamente à dados incompletos e relato seletivo dos desfechos. $^{(14-17)}$

Outros riscos como a utilização de dois tipos de interfaces, a heterogeneidade dos sujeitos e a análise restrita de respirações desencadeadas pela AEdi, aparentam um "alto risco" de viés na análise dos resultados publicados por Vignaux et al. (2013). ${ }^{(14)}$ Assim como a não descrição do tempo de washout, o uso de diversos tipos de interfaces e dispositivos de VM durante a aplicação das intervenções, classificaram o estudo de Ducharme-Crevier et al. de como "alto risco" de viés. ${ }^{(17)}$ 
Random sequence generation (selection bias)

Allocation concealment (selection bias)

Blinding of participants and personnel (performance bias)

Blinding of outcome assessment (detection bias)

Incomplete outcome data (attrition bias)

Selective reporting (reporting bias)

Other bias

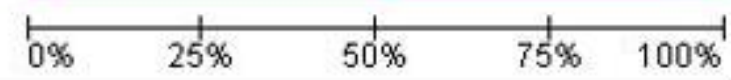

Low risk of bias

Unclear risk of bias

High risk of bias

Figura 2. Gráfico de análise do risco de viés: julgamento dos autores sobre cada item de risco de viés apresentado como porcentagem em todos os estudos incluídos.

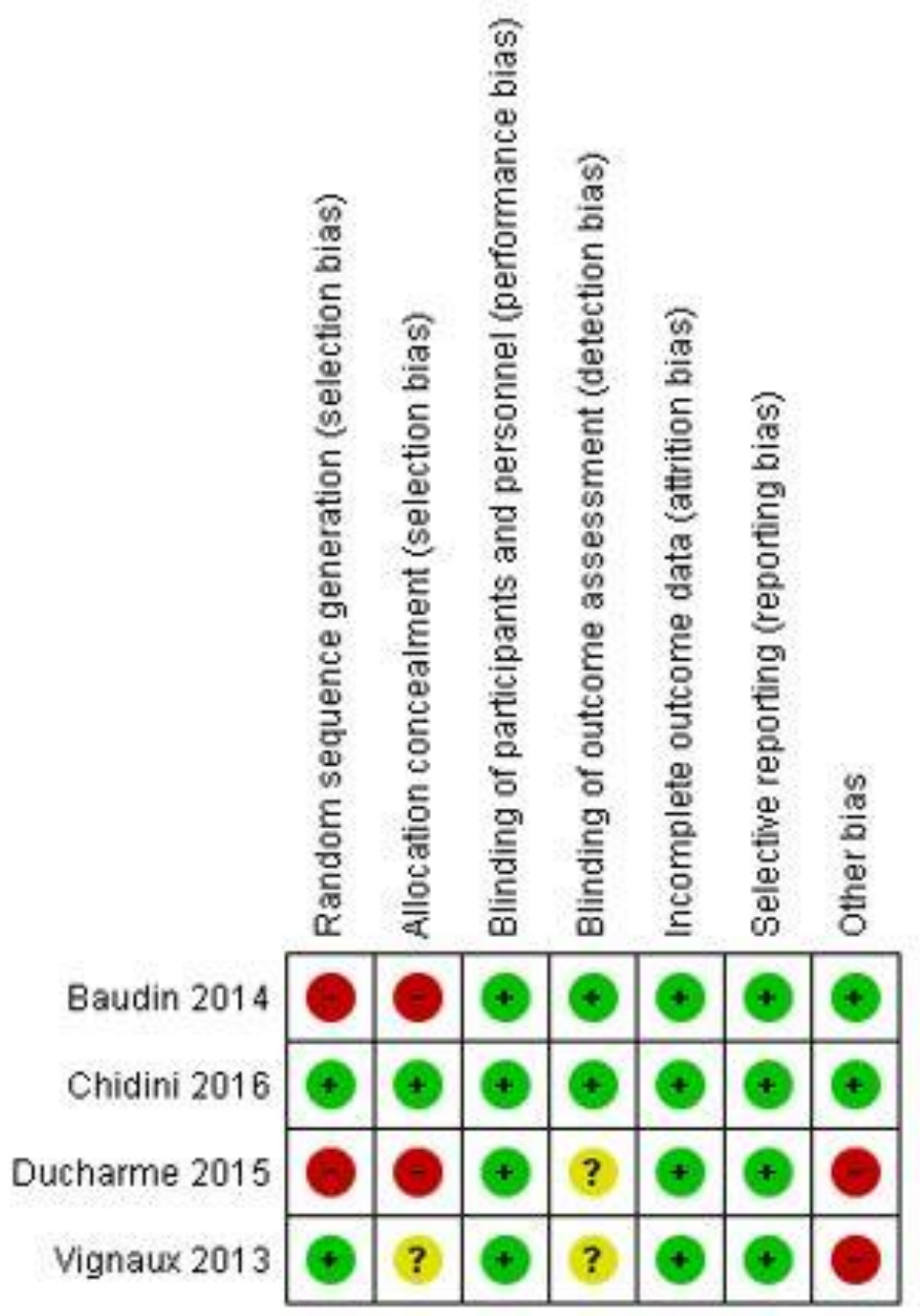

Figura 3. Resumo do risco de viés: análise dos julgamentos dos autores sobre cada item de risco de viés para cada estudo incluído 


\section{DISCUSSÃO}

Foram incluídos quatro estudos que analisaram a sincronia e os desfechos clínicos associados a aplicação da NIV NAVA em comparação a VNI Convencional durante o tratamento de crianças 39 crianças (elegíveis) com IRpA. ${ }^{(14-17)}$

Três estudos apresentaram diminuição significativa $(p<0,001)$ no IA durante a NIV NAVA, ${ }^{(14-16)}$ enquanto um estudo apresentou a diminuição na porcentagem de tempo gasto em assincronias durante a NIV NAVA $(p=0,05) .{ }^{(17)}$ Segundo os autores, a escolha desse tipo de variável se deu pela particular importância das assincronias inspiratórias e de ciclagem sobre a população pediátrica. Uma das desvantagens relacionadas, é a impossibilidade de comparar efeitos semelhantes obtidos de variáveis distintas, sendo que o IA expressa a porcentagem de eventos assincrônicos por minuto e não pelo o tempo cumulativo gasto em cada forma de assincronia. ${ }^{(10,17-19)}$

A relação entre a utilização da NIV NAVA e os desfechos clínicos foi inconclusiva entre os estudos. Enquanto Vignaux et al. (2013) relataram um aumento da pressão de pico inspiratória e pressão média das vias aéreas, ${ }^{(14)}$ Chidini et al. (2016) relataram resultados divergentes sobre as mesmas variáveis. ${ }^{(15)}$ Baudin et al. (2014) e Chidini et al. (2016), apresentaram resultados semelhantes no aumento da $\mathrm{FR}^{(15,16)}$ e do Vmin, ${ }^{(15)}$ enquanto Vignaux et al. (2013) não observaram alterações significantes sobre as mesmas variáveis. ${ }^{(14)} \mathrm{O}$ relato dos desfechos dessa revisão também foram inconclusivos com os descritos na literatura prévia, ${ }^{(8,11,12,20)}$ impedindo a confirmação da hipótese de correlação.

A utilização do método de seleção cruzada (cross-over) adotado pelos autores, ${ }^{(14-17)}$ impossibilita a correlação de uma intervenção à desfechos clínicos específicos (ex.: tempo de internação na UTI, tempo total de VM, risco de lesão pulmonar induzida pela VM e risco de mortalidade). Assim como o período de washout não padronizado pode interferir nas análises dos resultados devido ao efeito terapêutico remanescente desconhecido entre as intervenções. Limitações relacionadas ao pequeno tamanho das amostras com alta heterogeneidade entre os sujeitos de pesquisa, podem estar associados a fatores de confusão na análise dos resultados, diminuindo assim a validade interna e externa dos estudos. 
Os autores dessa revisão hipotetizam que tais limitações apresentadas nos estudos podem estar associadas ao alto custo relativo do dispositivo de interação neural, uma vez que a tecnologia NAVA é privada e patenteada pela Getinge Group@, e está disponível apenas nos equipamentos Servo iß, Servo n® e Servo uß. Estudos comparando a relação entre custo e efetividade da tecnologia são escassos e limitados a participantes adultos sob VMI. ${ }^{(21)}$

Parte da heterogeneidade dos sujeitos de pesquisa tem relação direta entre a faixa etária da população e a causa da IRpA. Sendo que, crianças mais jovens como as observadas no estudo de Baudin et al. (2014), ${ }^{(16)}$ são mais suscetíveis a desenvolverem IRpA por infecções virais, como por exemplo a bronquiolite viral aguda (BVA). ${ }^{(22)}$ Enquanto crianças com mediana de idade maior, como as observadas no estudo de Ducharme-Crevier et al. (2015), ${ }^{(17)}$ são mais suscetíveis a desenvolverem IRpA após os procedimentos cirúrgicos ou por infecções bacterianas (ex.: pneumonia, sepse, etc.). ${ }^{(23,24)}$ Podendo assim, apresentar alterações heterogêneas durante o tratamento da IRpA. 


\section{CONCLUSÕES}

1. Após a revisão sistemática dos quatro artigos elegíveis, concluímos que a assistência ventilatória não invasiva ajustada neuralmente (noninvasive neurally adjusted ventilatory assist) apresenta um menor índice de assincronias quando comparada à ventilação não invasiva convencional em pacientes pediátricos com insuficiência respiratória aguda;

2. Devido às limitações metodológicas, os desfechos clínicos referentes à maior eficácia nas trocas gasosas, média dos parâmetros ventilatórios, desconforto respiratório e uso de sedativos foram inconclusivos;

3. Sugerimos que novos ensaios clínicos randomizados, com amostra homogênea alocada em grupos distintos (controle e intervenção), utilizando equipamentos e interfaces equivalentes, assim como um maior tempo de follow-up dos participantes, serão necessários para estabelecer uma relação mais precisa entre o menor índice de assincronias e os desfechos clínicos em pacientes sob ventilação mecânica não invasiva. 


\section{REFERÊNCIAS}

1. Friedman ML, Nitu ME. Acute respiratory failure in children. Pediatr Ann. 2018;47(7):e26873.

2. Wolfler A, Raimondi G, Pagan de Paganis C, Zoia E. The infant with severe bronchiolitis: from high flow nasal cannula to continuous positive airway pressure and mechanical ventilation. Minerva Pediatr. 2018;70(6):612-22.

3. Abadesso $C$, Nunes $P$, Silvestre $C$, Matias $E$, Loureiro $H$, Almeida $H$. Non-invasive ventilation in acute respiratory failure in children. Pediatr Rep. 2012;4(2):e16.

4. Demaret P, Mulder A, Loeckx I, Trippaerts M, Lebrun F. Non-invasive ventilation is useful in paediatric intensive care units if children are appropriately selected and carefully monitored. Acta Paediatr. 2015;104(9):861-71.

5. Fauroux B, Lavis JF, Nicot F, Picard A, Boelle PY, Clément A, Vazquez MP. Facial side effects during noninvasive positive pressure ventilation in children. Intensive Care Med. 2005;31(7):965-9.

6. Gonzaga CS, Silva DC, Alonso CF, Oliveira CA, Torreão LA, Troster EJ. Noninvasive ventilation for acute respiratory failure in children - a systematic review. Einstein (Sao Paulo). 2011;9(1):90-4.

7. Al Otair HA, BaHammam AS. Ventilator- and interface-related factors influencing patientventilator asynchrony during noninvasive ventilation. Ann Thorac Med. 2020;15(1):1-8.

8. Suarez-Sipmann F; Acute Respiratory Failure Working Group of the SEMICYUC. New modes of assisted mechanical ventilation. Med Intensiva. 2014;38(4):249-60.

9. Colombo D, Cammarota G, Alemani M, Carenzo L, Barra FL, Vaschetto R, et al. Efficacy of ventilator waveforms observation in detecting patient-ventilator asynchrony. Crit Care Med. 2011;39(11):2452-7.

10. Sousa ML, Magrans R, Hayashi FK, Blanch L, Kacmarek RM, Ferreira JC. Predictors of asynchronies during assisted ventilation and its impact on clinical outcomes: the EPISYNC cohort study. J Crit Care. 2020;57:30-5.

11. Subira C, de Haro C, Magrans R, Fernandez R, Blanch L. Minimizing asynchronies in mechanical ventilation: current and future trends. Respir Care. 2018;63(4):464-78.

12. Lee J, Kim HS, Jung YH, Choi CW, Jun YH. Neurally adjusted ventilatory assist for infants under prolonged ventilation. Pediatr Int (Roma). 2017;59(5):540-4.

13. Higgins JP, Thomas J, Chandler J, Cumpston M, Li T, Page MJ, et al.; editors. Cochrane handbook for systematic reviews of interventions: version 6.0. Chichester: John Wiley \& Sons; 2019.

14. Vignaux L, Grazioli S, Piquilloud L, Bochaton N, Karam O, Levy-Jamet Y, et al. Patientventilator asynchrony during noninvasive pressure support ventilation and neurally adjusted ventilatory assist in infants and children. Pediatr Crit Care Med. 2013;14(8):e357-64.

15. Chidini G, De Luca D, Conti G, Pelosi P, Nava S, Calderini E. Early noninvasive neurally adjusted ventilatory assist versus noninvasive flow-triggered pressure support ventilation in pediatric acute respiratory failure: a physiologic randomized controlled trial. Pediatr Crit Care Med. 2016;17(11):e487-95. 
16. Baudin F, Pouyau R, Cour-Andlauer F, Berthiller J, Robert D, Javouhey E. Neurally adjusted ventilator assist (NAVA) reduces asynchrony during non-invasive ventilation for severe bronchiolitis. Pediatr Pulmonol. 2015;50(12):1320-7.

17. Ducharme-Crevier L, Beck J, Essouri S, Jouvet P, Emeriaud G. Neurally adjusted ventilatory assist (NAVA) allows patient-ventilator synchrony during pediatric noninvasive ventilation: a crossover physiological study. Crit Care. 2015;19(1):44.

18. Thille AW, Rodriguez P, Cabello B, Lellouche F, Brochard L. Patient-ventilator asynchrony during assisted mechanical ventilation. Intensive Care Med. 2006;32(10):1515-22.

19. Chao DC, Scheinhorn DJ, Stearn-Hassenpflug M. Patient-ventilator trigger asynchrony in prolonged mechanical ventilation. Chest. 1997;112(6):1592-9.

20. Chen C, Wen T, Liao W. Neurally adjusted ventilatory assist versus pressure support ventilation in patient-ventilator interaction and clinical outcomes: a meta-analysis of clinical trials. Ann Transl Med. 2019;7(16):382.

21. Hjelmgren J, Bruce Wirta S, Huetson P, Myrén KJ, Göthberg S. Health economic modeling of the potential cost saving effects of Neurally Adjusted Ventilator Assist. Ther Adv Respir Dis. 2016;10(1):3-17.

22. Nair H, Nokes DJ, Gessner BD, Dherani M, Madhi SA, Singleton RJ, et al. Global burden of acute lower respiratory infections due to respiratory syncytial virus in young children: a systematic review and meta-analysis. Lancet. 2010;375(9725):1545-55.

23. Balcells Ramirez J, Lopez-Herce Cid J, Modesto Alapont V; Grupo de Respiratorio de la Sociedad Espanola de Cuidados Intensivos Pediatricos. [Prevalence of mechanical ventilation in pediatric intensive care units in Spain]. An Pediatr (Barc). 2004;61(6):533-41. Spanish.

24. Farias JA, Fernandez A, Monteverde E, Flores JC, Baltodano A, Menchaca A, et al.; LatinAmerican Group for Mechanical Ventilation in Children. Mechanical ventilation in pediatric intensive care units during the season for acute lower respiratory infection: a multicenter study. Pediatr Crit Care Med. 2012;13(2):158-64. 


\begin{abstract}
Introduction: The acute respiratory failure is the most prevalent comorbidity in children hospitalized in the intensive care unit in Brazil and worldwide, and the use of noninvasive mechanical ventilation is considered for the treatment of mild and moderate cases of the disease. Conventional flow or pressure trigger modes during noninvasive ventilation have a higher rate of asynchronies when compared to the trigger mode synchronized with the electrical activity of the diaphragm. Based on previous studies described in the literature, the hypothesis of the present study is that there is evidence of lower rate of asynchronies and favorable clinical outcomes related to the noninvasive neurally adjusted ventilatory assist mode when compared to the use of conventional noninvasive ventilation in pediatric patients with acute respiratory failure. Purpose: To compare the asynchrony index and clinical outcomes presented during the use of non-invasive neurally adjusted ventilatory assist vs. conventional noninvasive ventilation in the treatment of acute respiratory failure in the pediatric population. Methods: This is a systematic review of clinical trials conducted between April and May 2020 in the electronic databases Cochrane Library, Embase, LILACS, PubMed/MEDLINE, Scopus and Web of Science. Results: A total of 184 studies were found, four of which were eligible for qualitative synthesis. The sample analyzed 39 participants, aged between 35 days and 15 years with male predominance $(61.5 \%)$. The primary outcome analyzed in three of the four studies was the significant decrease ( $p$ $<0.001$ ) in the asynchrony index during non-invasive neurally adjusted ventilatory assist compared to conventional noninvasive ventilation. However, the hypothesis of correlation between the improvement of synchrony and favorable clinical outcomes was inconclusive due to methodological limitations of the studies. Conclusions: After systematic review, we conclude that non-invasive neurally adjusted ventilatory assist decreases the asynchrony index when compared to conventional noninvasive ventilation in pediatric patients with acute respiratory failure. However, the other clinical outcomes present inconclusive results, requiring further studies with different methodological formats for confirmation.
\end{abstract}

PROSPERO ID: 181785

Keywords: Noninvasive ventilation; Interactive ventilatory support; Infant; Child, preschool; Child 